\title{
Structural biology contributions to the discovery of drugs to treat chronic myelogenous leukemia
}

\section{Sandra W. Cowan-Jacob, 'Gabriele Fendrich, Andreas Floersheimer, Pascal Furet, Janis Liebetanz, Gabriele Rummel, Paul Rheinberger, Mario Centeleghe, Doriano Fabbro and Paul W. Manley}

Novartis Institutes for Biomedical Research, Basel, , Switzerland. E-mail: sandra.jacob@novartis.com

\section{Supplementary material}

Difference Fouriers for ligands in each of the crystal structures presented in the article.

Figure S1. Difference Fourier contoured at $2.5 \sigma$ (blue mesh) for imatinib (yellow carbon, blue nitrogen, red oxygen) in complex with human Abl kinase.

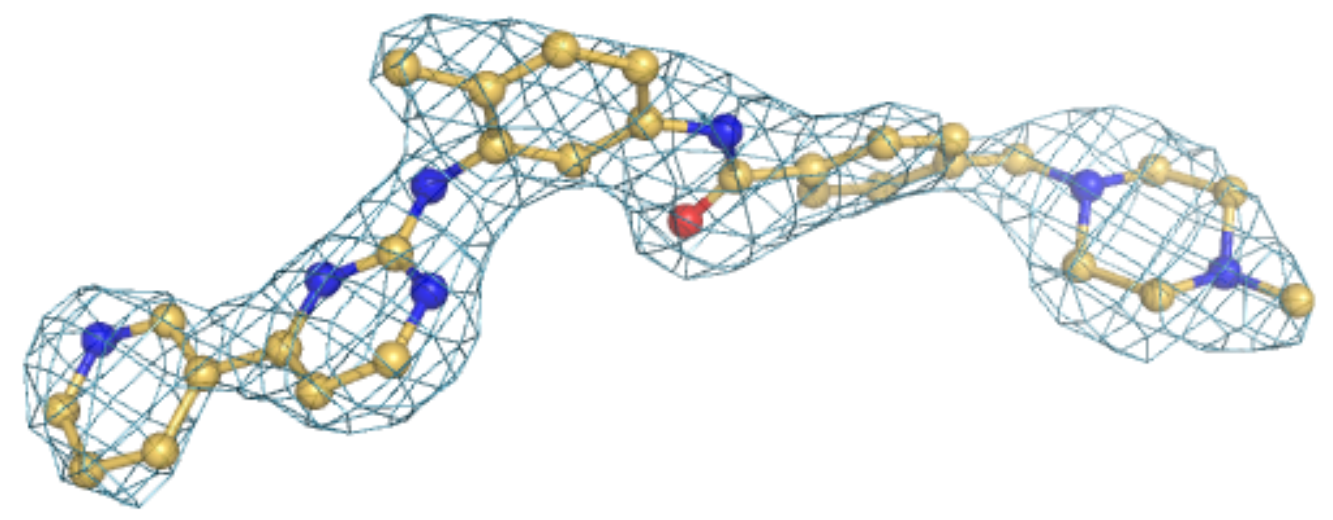

Figure S2. Difference Fourier contoured at $2.5 \sigma$ (blue mesh) for NVP-AEG082 (yellow carbon, blue nitrogen, red oxygen) in complex with Abl kinase.

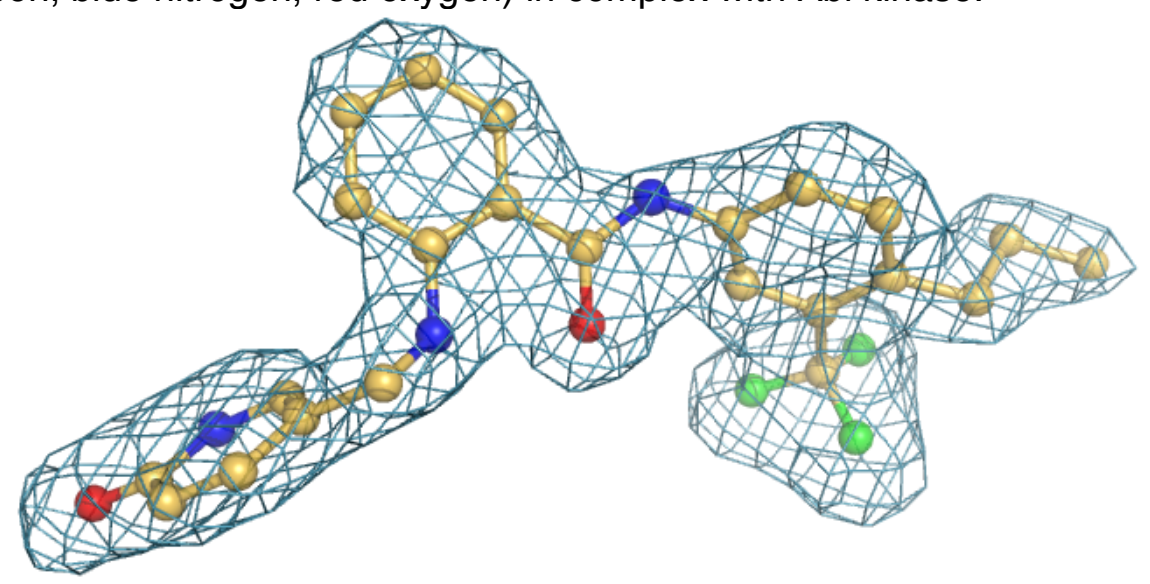


Figure S3. Difference Fourier contoured at $2.5 \sigma$ (blue mesh) for NVP-AFN941 (yellow carbon, blue nitrogen, red oxygen) in complex with Abl kinase.

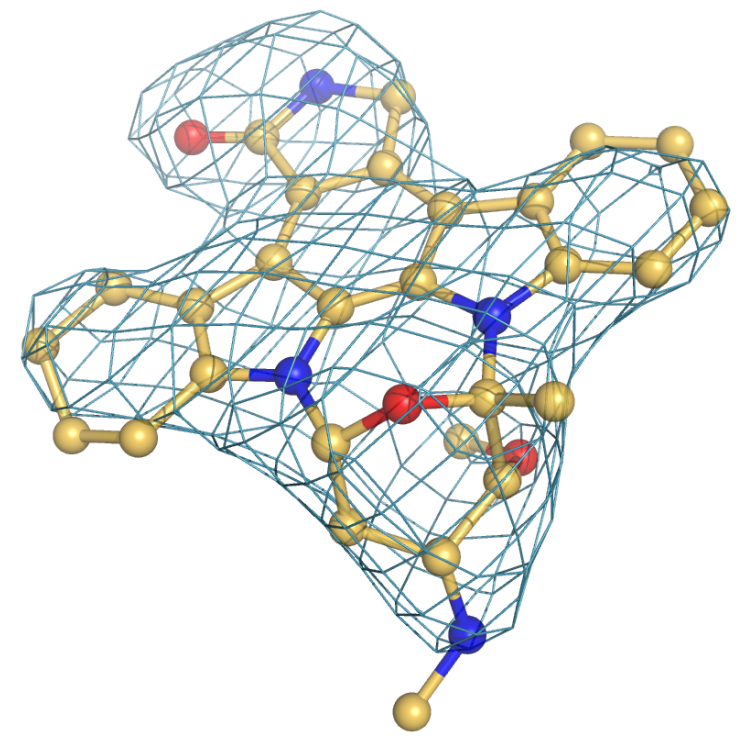

Figure S4. Difference Fourier contoured at $2.5 \sigma$ (blue mesh) for NVP-AFG210 (yellow carbon, blue nitrogen, red oxygen, green fluorine) in complex with Abl kinase.

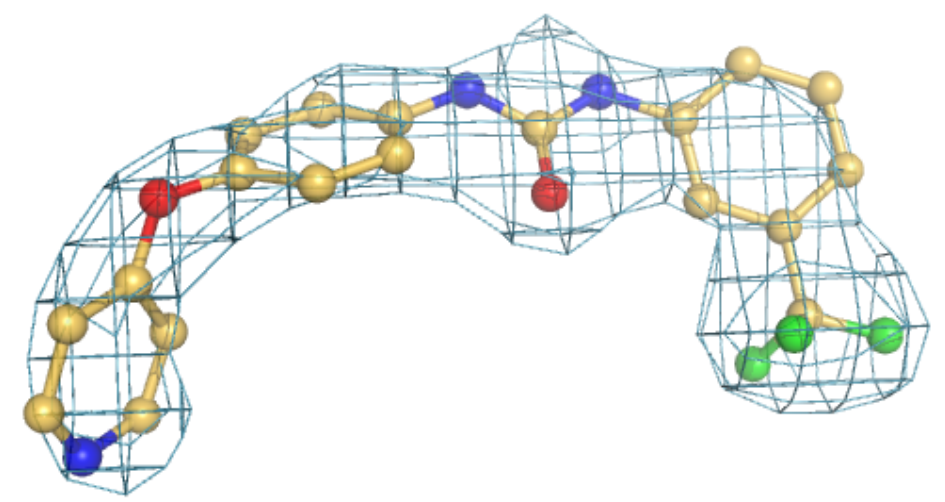

Figure S5. Difference Fourier contoured at $2.5 \sigma$ (blue mesh) for PD180970 (yellow carbon, blue nitrogen, red oxygen, green fluorine, magenta chlorine) in complex with Abl kinase.

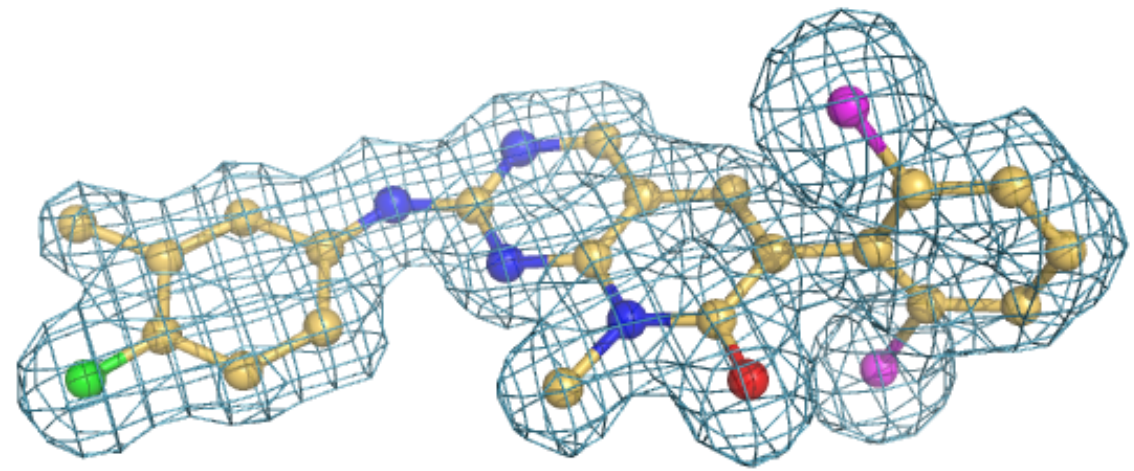

